\title{
Fluktuationen der Fischfauna im Elbe-Ästuar als Indikator für ein gestörtes Ökosystem
}

\author{
Angela Köhler \\ Zoologisches Institut und Zoologisches Museum der Universität Hamburg; \\ Martin-Luther-King-Platz 3, 2000 Hamburg 13, Bundesrepublik Deutschland
}

\begin{abstract}
Fluctuations of the ichthyo-fauna in the Elbe estuary: an indicator for a disturbed ecosystem. During 1978, 22 fish species (in particular Anguilla anguilla L., Platichthys flesus L., Osmerus eperlanus L., Gasterosteus aculeatus L., Lampetra fluviatilis L., Alosa fallax [Lacepede], Gymnocephalus cernua L., Clupea harengus L. and Sprattus sprattus L.), sampled at the intake of the cooling system in the nuclear power plant at Brunsbüttel (Elbe estuary), were analyzed for quantities and size distribution. The data obtained were correlated to abiotic factors, such as water temperature, water outflow from the upper Elbe river, salinity and oxygen content. Spawning times and seasonal migrations of the fish species investigated corresponded to appropriate temperatures of the Elbe water. The diversity of fish species from the cooling water proved to be representative for the ichthyo-fauna of this particular estuarine area. At least 190 tons of fish per year were estimated to be annihilated by the suction of cooling water into the nuclear power plant. In spite of the progressive development of regional industries and the increasing discharges of cooling water, temperature in the estuary has remained largely unaffected up till now. The oxygen content of the heavily polluted lower Elbe river, however, has become mainly dependent on the amount of tresh water flowing from the upper Elbe river. Up to 1978, oxygen levels of 80-90\% were recorded only in the mouth of the Elbe, thus meeting the requirements for the continuous occurrence of fish species typical for this estuarine area. Varying oxygen concentrations downstream of Hamburg and at Brunsbüttel are considered to be responsible for migrations of certain fish species (mainly flounder and smelt) between the Brunsbüttel region and their habitats further upstream which were occupied before the process of industrialisation initiated. This was reflected by the size of the samples taken from various fishes. During an eventual hazard of the industrial filter plants at Brunsbüttel in May 1978 an extreme oxygen depletion occurred for nearly 2 weeks. This event coincided with the death of certain age groups of fish.
\end{abstract}

\section{EINLEITUNG}

Das Mündungsgebiet der Elbe ist nach der Industrialisierung des Unterelberaumes eine Region, in welcher während des ganzen Jahres eine kommerziell lohnende Elbfischerei noch möglich ist. Extreme Sauerstoffdefizite unterhalb Hamburgs durch die Einleitung häuslicher Abwässer limitieren die flußaufwärts gerichtete Verbreitung typischer Fischarten des Elbe-Astuars (Wilkens \& Köhler, 1977; ARGE Elbe, 1977-79). Schadstoffeinleitungen (Schwermetalle, chlorierte Kohlenwasserstoffe) der Industrieansiedlungen der Unterelbe und aus der DDR führen zu schweren Gesundheitsschädigungen einiger Elbfischarten (Köhler, 1979; Köhler \& Hölzel, 1980).

Die abiotischen Faktoren Wassertemperatur, Oberwasserabfluß und Sauerstoffgehalt bestimmen das natürliche, starken Fluktuationen unterliegende ichthyologische 
Bild der Unterelbe zu verschiedenen Jahreszeiten (Ehrenbaum, 1896; Schnackenbeck, 1928; Bauch, 1958; Lillelund, 1961; Tesch, 1977; Wilkens \& Köhler, 1977). Ziel der vorliegenden Untersuchung ist es, den Einfluß der erwähnten Faktoren auf das Vorkommen der Fischarten in dem gestörten Ókosystem Unterelbe zu ermitteln.

Die Möglichkeit zu einer kontinuierlichen Fischprobennahme über einen Zeitraum von eineinhalb Jahren bot sich aufgrund einer Untersuchung der Bundesforschungsanstalt für Fischerei (Hamburg) im Kernkraftwerk Brunsbüttel. Die Untersuchung wurde eingeleitet, da wegen eines extrem hohen Fischanfalles im Kühlwasser Zweifel an der Funktionstüchtigkeit der dem Kühlwassereinlaufbauwerk vorgelagerten Fischscheuchanlage bestanden.

Unter Berücksichtigung der hydrologischen Messungen in der Elbe im Untersuchungsjahr wurde überprüft, welche der abiotischen Faktoren die Artenzusammensetzung und die Schwankungen der Anzahl und der Größenklassen der im Kernkraftwerk Brunsbüttel angesogenen Fischarten bestimmen. Gleichzeitig wurde an Hand der Literaturdaten zur Verbreitung und zum Verhalten der Fischfauna untersucht, ob das sich im Kernkraftwerk Brunsbüttel ergebende Bild für das ichthyologische Geschehen im ElbeAstuar repräsentativ ist.

\section{MATERIAL UND METHODEN}

Die Fischprobenentnahme der vorliegenden Untersuchungen wurde am Fischrechen des Kühlwassersystems des Kernkraftwerkes Brunsbüttel (Stromkilometer 693; vgl. Lageskizze in Köhler \& Hölzel, 1980, p. 405) durchgeführt. Das Kühlwasser wird von zwei Einsaugkanälen von ca. 9,2 $\mathrm{m}^{2}$ Querschnitt in 5-7 m Wassertiefe in ca. $100 \mathrm{~m}$ Abstand vom Ufer der Elbe entnommen. Die Abstände der Rechenzähne, von denen die Fische aus dem Kühlwasser ferngehalten werden sollen, betragen am Grobrechen 40 $\mathrm{mm}$ Abstand und am Feinrechen $5 \mathrm{~mm}$ Gitterabstand. Am Grobrechen wurden nur Brassen bis $50 \mathrm{~cm}$ Länge gefangen, so daß die Probenahme der übrigen Fischarten am Feinrechen erfolgte.

Die Probenahme im Kernkraftwerk Brunsbüttel wurde, soweit dies aus organisatorischen Gründen möglich war, 14tägig durchgeführt. Untersucht wurde die Fischmenge, die während der Dauer von 24 Stunden mit dem Kühlwasser angesogen wurde. Da die Probenahmezeit 2 Tiden einschloß, konnten von Ebbe und Flut bewirkte Einflüsse auf die Fischverbreitung vor dem Einlaufbauwerk bei der Auswertung der Daten vernachlässigt werden. Die Probenahme dauerte jeweils von 6 Uhr morgens bis 6 Uhr morgens am folgenden Tage. Auf diese Weise wurden auch Fische mit unterschiedlichen Tagesaktivitätsphasen in den Proben erfaßt. Insgesamt wurden in einem Zeitraum von 11/2 Jahren 32 Proben genommen, von denen 23 - entsprechend einem Jahreszyklus - in der vorliegenden Arbeit zur Auswertung kamen.

Für die qualitative und quantitative Analyse der Fischproben wurden die Fischarten bestimmt und vor Ort gemessen und gewogen. Ergaben die 24 -Stunden-Fänge große Fischmengen, wurden die seltener auftretenden Arten aussortiert; von den zahlreich bis massenhaft vorkommenden Arten wurden repräsentative Unterproben (bis $30 \mathrm{~kg}$ ) genommen.

Die Menge des der Elbe bei Brunsbüttel entnommenen Kühlwassers schwankte im Untersuchungszeitraum zwischen 8000 und $130000 \mathrm{~m}^{3} / \mathrm{h}$. Während der meisten Probe- 
nahmen konnte jedoch erreicht werden, daß eine Kühlwassermenge von $120000 \mathrm{~m}^{3} / \mathrm{h}$ für 24 Stunden angesogen wurde. Um eine repräsentative Aussage für die Situation des Vollbetriebes des Kernkraftwerkes zu bekommen, wurden sämtliche Fischarten auf die normalerweise entnommene Kühlwassermenge von $130000 \mathrm{~m}^{3} / \mathrm{h}$ hochgerechnet. Bei der Interpolation der vernichteten Fischmengen auf das gesamte Jahr wurden Phasen erhöhter Aktivität, wie in Laichzeiten, sommerlichen Nahrungswanderungen und Blankaalwanderungen, gesondert berücksichtigt.

Die für die ökologische Auswertung notwendigen Daten über die Wassertemperaturen, den Oberwasserabfluß, die Sauerstoffgehalte und die Stickstoffverbindungen des Elbwassers entstammen den täglichen und wöchentlichen Untersuchungen der Wassergütestelle Hamburg (vgl. ARGE Elbe, 1978). Zur Beschreibung der Situation im Jahre 1978 wurden in dem Begriff Gesamtstickstoff die quantitativen Angaben von $\mathrm{NH}_{4}{ }^{+}$. $\mathrm{NO}_{2}{ }^{-}$und $\mathrm{NO}_{3}{ }^{-}$, sowie des organisch gebundenen Stickstoff zusammengefaßt.

Die reststrombedingte Transportzeit (flushing time) ist die Zeit, die ein Wasserteilchen unabhängig von Tideeinflüssen für die Zurücklegung der Strecke von Darchau (Stromkilometer 535) bis Brunsbüttel (Stromkilometer 693) benötigte. Sie berechnet sich aus der mittleren Strömungsgeschwindigkeit bei einem mittleren wöchentlichen Oberwasserabfluß und den durchschnittlichen Flußquerschnitten (mündliche Mitteilung von Maier-Reimers, Max-Planck-Institut für Meteorologie, Hamburg).

\section{ERGEBNISSE}

\section{Wasserqualität der Unterelbe}

Die Wasserqualität der Unterelbe wird heute aufgrund der starken Belastung durch industrielle und häusliche Abwässer in hohem Maße von der Menge des zufließenden Süßwassers (Oberwasser) und vom Eindringen $\mathrm{O}_{2}$-reichen Seewassers bestimmt. In Zeiten niedriger Oberwasserabflüsse übt die starke organische Belastung, besonders des Hamburger Raumes, einen enormen Einfluß auf die Sauerstoffbedingungen des Flußabschnittes unterhalb Hamburgs aus, so daß extreme Sauerstoffdefizite mehrmals im Jahr das Leben dieser Zone bedrohen (Köhler, 1980; Köhler \& Hölzel, 1980). Während länger andauernder Perioden hoher Wasserzuflüsse tritt eine erhebliche Verbesserung der Sauerstoffverhältnisse neben einer allgemeinen Verdünnung aller Schadstoffe unterhalb Hamburgs ein (Wilkens \& Köhler, 1977; Köhler \& Hölzel, 1980).

Die hohen Oberwasserzuflüsse über $1000 \mathrm{~m}^{3} / \mathrm{s}$, gemessen bei Darchau, erreichten bereits nach 7-10 Tagen die Mündungsregion der Elbe unter Berücksichtigung der reststrombedingten Transportzeit (Köhler, 1980) und bewirkten erwartungsgemäß zunächst bei Wedel und mit einiger Verzögerung bei Brunsbüttel eindeutige Sauerstoffverbesserungen (Abb. 1). Besonders deutlich war der Zusammenhang zwischen Oberwasserzufuhr und dem Sauerstoffgehalt Ende Mai/Anfang Juni sowie Anfang August und im September. Die erhöhte Wasserzufuhr führte ebenfalls stets zu einer Abnahme aller gemessenen Stickstoffverbindungen $\left(\mathrm{NH}_{4}{ }^{+}, \mathrm{NO}_{3}{ }^{-}\right.$, Gesamtstickstoff $\left.=\mathrm{N}_{\mathrm{G}}\right)$.

$\mathrm{Zu}$ Zeiten von Niedrigwasserabflüssen benötigten die bei Darchau registrierten Zuflüsse ca. 35 Tage, um in der Elbmündung einzutreffen, so daß die "Schmutzfracht" der Unterelbe über einen längeren Zeitraum kaum verdünnt wurde. Dies zeigte sich in 


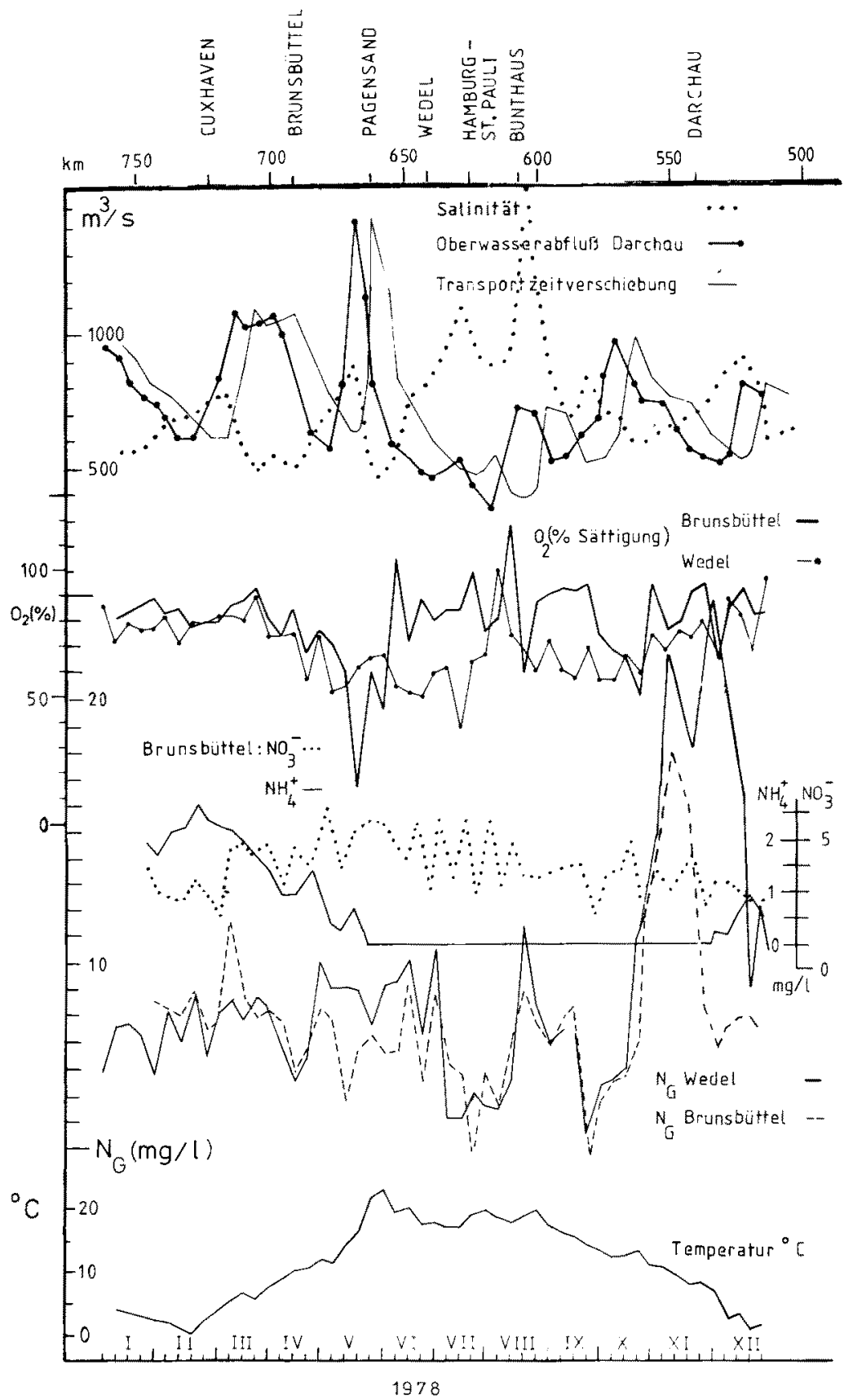

Abb. 1. Wassertemperatur, Stickstoffverbindungen $\left(\mathrm{NH}_{4}{ }^{+}\right.$und $\left.\mathrm{NO}_{3}{ }^{-}\right)$und Salinität bei Brunsbüttel, Gesamtstickstoffgehalte und Sauerstoffsättigung (in \%) bei Wedel und Brunsbüttel und der Oberwasserabfluß (Darchau) unter Berücksichtigung der reststrombedingten Transportzeit (nach Daten der ARGE Elbe, 1978) 
den Meßwerten der ARGE Elbe (1978) in einem periodischen Ansteigen der Gesamtstickstoffmengen (Abb. 1). Der genaue Jahresverlauf der Beziehungen zwischen Oberwasserabfluß, Sauerstoffgehalt und dem temperaturabhängigen Jahresgang der biologischen Oxidation von Stickstoffverbindungen ist anderweitig ausführlich dargestellt (Köhler, 1980).

Im Mai 1978 trat im Raum Brunsbüttel ein extremes Sauerstoffminimum auf, das vom 19. 5.-2. 6. $78 \mathrm{zu}$ einem der größten Fischsterben seit 30 Jahren führte. Derartige Sauerstoffdefizite konnten bis zu diesem Jahr in der Elbmündung im Frühjahr und Sommer nicht festgestellt werden. Die Beobachtung der Meßwerte im April und Anfang Mai lieferte keinerlei Hinweise auf ein im Mai bevorstehendes Sauerstoffdefizit bei Wedel oder Brunsbüttel. Lediglich der Beginn der Nitrifikationsprozesse bei einem Temperaturanstieg auf $10^{\circ} \mathrm{C}$ deutete sich in der Abnahme der $\mathrm{NH}_{4}{ }^{+}$-Werte und der Zunahme der $\mathrm{NO}_{3}{ }^{-}$-Werte an. Am Untersuchungstag des 24.5. 78 wurde bei Brunsbüttel ein plötzlich auftretendes und für diese Region ungewöhnliches Sauerstoffminimum von nur $15 \%$ der Sättigung gemessen. Bei einer Wassertemperatur von $15,2{ }^{\circ} \mathrm{C}$ konnte $\mathrm{NH}_{4}{ }^{+}$ nicht vollständig zu Nitrat oxidiert werden. Trotz intensiver Sonneneinstrahlung in diesem Zeitraum wurde der Sauerstoffverlust von der entsprechend hohen biogenen Sauerstoffproduktion durch photosynthetische Prozesse nicht kompensiert. Erst am 31. 5. 78 fand im Bereich Brunsbüttel eine vollständige Nitrifikation statt, erkennbar an den konstant hohen $\mathrm{NO}_{3}{ }^{-}$-Werten und der erneuten Abnahme der $\mathrm{NH}_{4}{ }^{+}$-Werte. Der Sauerstoffgehalt des Elbwassers hatte sich bei Wassertemperaturen von $20,5^{\circ} \mathrm{C}$ auf $59 \%$ der Sättigung $(5,2 \mathrm{mg} / \mathrm{l})$ verbessert. Diese Sauerstoffzunahme war auf eine zu diesem Zeitpunkt bei Brunsbüttel eintreffende hohe Oberwasserzufuhr bis über $1400 \mathrm{~m}^{3} / \mathrm{s}$, die 19 Tage lang andauerte, zurückzuführen (Abb. 1). Wie sich auch in den täglichen Sauerstoffmessungen bei Cuxhaven zeigte (ARGE Elbe, 1978), wurde infolge der Tidebewegung ein relativ großer Elbabschnitt, der bei Flut elbaufwärts bis Brokdorf (Stromkilometer 685) und bei Ebbe bis zum Neufelder Watt (Stromkilometer 725) reichte, von dem Sauerstoffdefizit erfaßt.

\section{Wichtigste Nutzfischarten}

Den Fischarten Flunder (Platichthys flesus L.), Stint (Osmerus eperlanus L.) und Aal (Anguilla anguilla L.) dient die Unterelbe für einen längeren Lebensabschnitt als Aufwuchs- und Nahrungsbiotop. Die Faktoren Temperatur, Oberwasserzufluß und Sauerstoffgehalt wirkten sich unterschiedlich auf die Fluktuationen im Fischvorkommen bei Brunsbüttel aus. Während die Wassertemperatur in erster Linie das Auftreten unterschiedlicher Größenklassen der genannten Fischarten in den Proben beeinflußte, zeigten Sauerstoffgehalt und Oberwasserabfluß eindeutige Auswirkungen auf das quantitative Vorkommen im Untersuchungsgebiet während eines Jahres.

Die Jungflundern wandern aus den Laichgebieten in der südlichen Nordsee mit einer Länge von 1-2 cm in die Elbmündung ein. Im Süß- und Brackwasser verbringen sie die ersten Lebensjahre.

Die Anzahl der Flundern schwankte in den Kühlwasserproben während des Untersuchungszeitraumes zum Teil erheblich (Abb, 2). Diese Veränderungen können als Hinweis interpretiert werden, daß die Flunderpopulation im Elbe-Ästuar eine starke 
Tendenz zu Wanderungen zeigte. Hoh e Oberwa s s e r a bf l üs s e bewirkten unter Berücksichtigung der reststrombedingten Transportzeit $S$ a uers t of f verbesser u n g e n, zunächst bei Wedel (Stromkilometer 642), später bei Brunsbüttel (Stromkilometer 693) (Abb. 1). Während des Zeitraumes verbesserter Sauerstoffbedingungen bei Wedel war stets ein bemerkenswerter Anstieg der Flunderzahlen in den Proben bei Brunsbüttel zu beobachten, der nur mit einer erhöhten Wanderaktivität der Flundern als Reaktion auf den Sauerstoffanstieg in ihrem ehemaligen Wohngebiet zu erklären ist. Besonders deutlich war dieser Einfluß, wenn die Oberwasserzuflüsse $900 \mathrm{~m}^{3} / \mathrm{s}$ überschritten hatten (Abb. 2). Bereits im Januar deutete sich diese Wechselwirkung zwischen den Wanderungen und den Sauerstoffgehalten bei Wedel an, wurde in den Monaten März und April klarer und trat im Mai und August am deutlichsten hervor.

In Zeiten einer Verschlechterung der Sauerstoffbedingungen unterhalb Hamburgs während niedriger Wasserzuflüs e aus der oberen Elbe

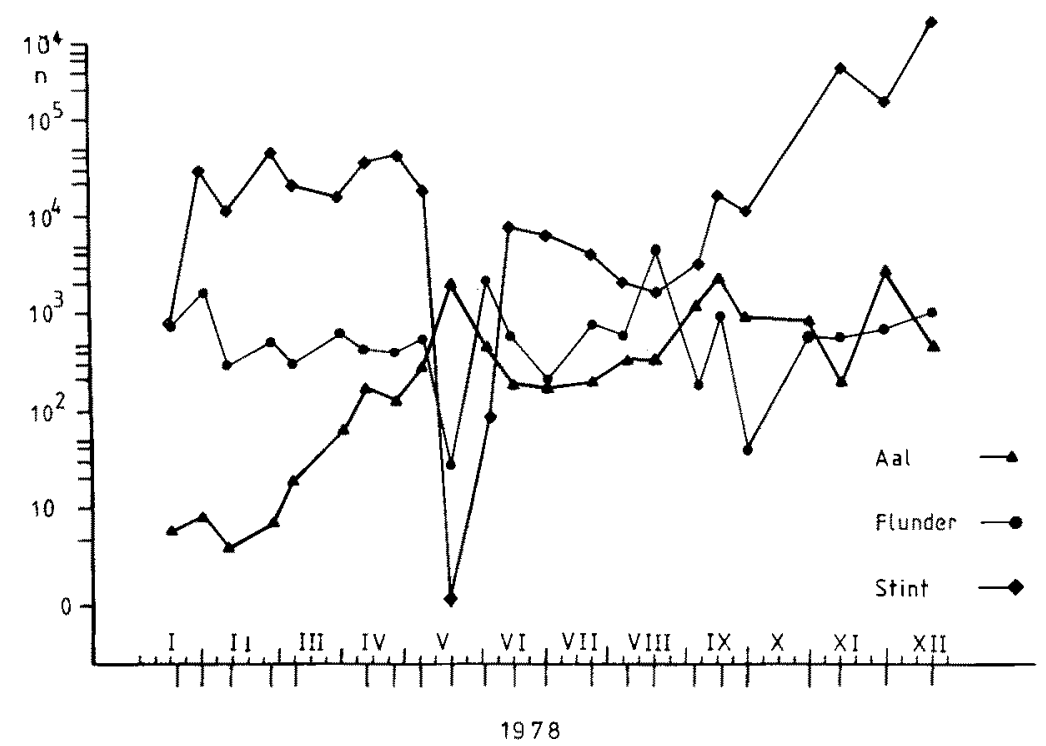

Abb. 2. Gesamtstückzahlen ( $\log n$ ) der Flunder (Platichthys flesus), des Stintes (Osmerus eperlanus) und des Aales (Anguilla anguilla) bei einer Kühlwasserentnahme von $130000 \mathrm{~m}^{3} / \mathrm{h}$ über eine Dauer von 24 Stunden im Kernkraftwerk Brunsbüttel

konnte wiederum ein wenn auch geringerer Anstieg der Flunderzahlen bemerkt werden. Dies wurde Ende Juli und Ende September in den Proben deutlich (Abb. 2). Vermutlich wanderten die Flundern bei sinkendem $\mathrm{O}_{2}$-Gehalt elbabwärts in den Raum Brunsbüttel, den sie bei weiteren $\mathrm{O}_{2}$-Abnahmen Anfang Oktober ebenfalls verließen.

Während des Sauerstoffminimums bei Brunsbüttel im Mai 1978 konnten nur 29 Flundern in den Kühlwasserproben gezählt werden. Die beträchtliche Oberwassermenge vom 14. 5. 1978 an leitete die bereits erwähnte Regeneration der Sauerstoffverhältnisse in der Elbmündung ein, die sich in einem extremen Anstieg rückwandernder Flundern in der Probe vom 7. 6. 1978 wiederspiegelte.

Im Gegensatz zur Flunder bewohnt der Stint das Pelagial des Elbe-Ästuars. Aus der 
Flußmündung ziehen die Stinte ungefähr Mitte März elbaufwärts zu ihren Laichplätzen. Der Stintaufstieg erfolgte bis in die sechziger Jahre stets innerhalb weniger Tage, erkennbar an einer rapiden Abnahme der Fangerträge im Unterlauf der Elbe und einem ebenso raschen Anstieg im flußauf gelegenen Elbgebiet (Lillelund, 1961).

Die Hauptlaichzeit vom 2, 3.-ca. 14.4., über eine Mindestdauer von 44 Tagen also, war von Oberwasserabflüssen von $680-1068 \mathrm{~m}^{3} / \mathrm{s}$ begleitet. Bei niedrigeren Oberwasserzuflüssen wuchsen die Stintzahlen Anfang März und Mitte April erheblich in den Proben an, so daß vermutet werden kann, daß sich die einwandernden Stintschwärme bei Brunsbüttel stauten (Abb. 2). Sowie die Abflüsse eine Höhe von $1000 \mathrm{~m}^{3} / \mathrm{s}$ in der 2 . Märzwoche erreicht hatten, sanken die Stintzahlen zunächst um ca, die Hälfte auf 21428 Stück/24 h und bei andauernden hohen Wasserzuflüssen auf 15812 Stück/24 h in den Proben ab. Im weiteren Jahresverlauf ließ sich eine Beziehung zwischen der Individuenzahl und den Sauerstoffgehalten im Untersuchungsgebiet unter extremen Bedingungen, d. h. bei Sauerstoffwerten unter $60 \%$ der Sättigung, herstellen. So führte das Sauerstoffminimum von nur $15 \%$ Sättigung am Probenahmetag des 24. 5. zu einem völligen Verschwinden der Stinte aus dem Kühlwasser des Kernkraftwerkes Brunsbüttel. Ein Teil des Stintbestandes wurde wahrscheinlich von dem Fischsterben vom 19. 5.-2. 6. erfaßt. Als die Sauerstoffwerte Anfang Juni wieder auf $45 \%$ der Sättigung gestiegen waren, konnten nur 82 Stinte im Kühlwasser gezählt werden. Erst nachdem hohe Oberwasserzuflüsse zu einer Sauerstoffverbesserung auf mehr als $70 \%$ geführt hatten, kehrten langsam wieder Stinte in den Kühlwassereinsaugbereich zurück (Abb. 2).

Im Spätsommer wurden die Beziehungen zwischen dem oberwasserabhängigen Sauerstoffgehalt bei Brunsbüttel und der Stinthäufigkeit wiederum deutlich. Niedrige $\mathrm{O}_{2}$-Gehalte unter $60 \%$ der Sättigung (Abb. 1) hatten offensichtlich eine Abnahme der Stinte bei Brunsbüttel im August und vermutlich auch im Oktober, wie sich am Monatsanfang andeutete, bei nur $53 \%$ Sauerstoffsättigung zur Folge (Abb. 2).

Im Elbe-Astuar kommen verschiedene Entwicklungsstadien des Aales im Wechsel mit den Jahreszeiten vor. Erst bei einer Länge von mehr als $14 \mathrm{~cm}$ wurden Aale im bereits pigmentierten Stadium von den Feinrechen im Kühlwassereinlaufbauwerk erfaßt. Da während des Untersuchungszeitraumes im April massenhaft Glasaale (Länge: $7-11 \mathrm{~cm}$ ) an den Elbufern beobachtet werden konnten, ist anzunehmen, daß große Mengen der kleinen Glasaale mit dem Kühlwasser den Reaktor passiert haben. Jungaale, Gelbaale und Blankaale wurden in großen Mengen mit dem Kühlwasser angesogen und von den Rechen zurückgehalten.

Während des bereits mehrfach erwähnten extremen Sauerstoffminimums im Mai 1978 zeigten die Aale eine dem Flunder- und Stintverhalten völlig entgegengesetzte Reaktionsweise. Sie wurden in für die Jahreszeit extrem hohen Stückzahlen (bis 1881 Stück/24h Kühlwasserentnahme am 24.5. 78), also 5 Tage nach dem Einsetzen des großen Sterbens, angesogen (Abb. 2 und 5). Sie waren offenbar weder aus dem betroffenen Gebiet abgewandert, noch bereits zu Beginn des Sauerstoffminimums verendet. Bei der Probenanalyse fiel auf, daß alle Aale apathisch oder nahezu bewegungslos waren.

Im weiteren Jahresverlauf zeigte sich, daß durch niedrige Oberwasserzuflüsse nach unten schwankende Sauerstoffgehalte nachlassende Aalzahlen zur Folge hatten, wie sich in den Proben Anfang Oktober und Mitte November beobachten ließ (Abb. 1 und 2).

Zufriedenstellende Sauerstoffverhältnisse bei Werten über $80 \%$ Sättigung, die 
jeweils als eine Folge einer im Raum Brunsbüttel eintreffenden erhöhten Oberwasserzufuhr beobachtet werden konnten, zogen einen Anstieg der Aalzahlen im Untersuchungsgebiet nach sich. Dieser Zusammenhang zeigte sich eindeutig in den Septemberproben und in der letzten Novemberprobe. In dieser Probe wurden auch die Blankaale aus der oberen Elbe und deren Seitengewässern miterfaßt, die nach der wanderungsauslösenden "Hoch"-Süßwasserzufuhr und niedrigen Wassertemperaturen (Tesch, 1977) nunmehr bei bereits nachlassendem Oberwasser die Elbmündung erreicht hatten. Durch die flußaufwärtsgerichtete Zuwanderung von Gelbaalen regenerierte sich im Herbst das Gelbaalvorkommen vor Brunsbüttel nach dem Fischsterben im Mai 1978. Während der gesamten Blankaalwanderung lagen Hamenfischer vor dem Kühlwassereinlaufbauwerk auf ihren Jahrhunderte alten Fangplätzen.

Die im Kernkraftwerk Brunsbüttel über das Auftreten der Flunder gewonnenen Daten verdeutlichen, wie die Was s ert em per a t u r das Vorkommen unterschiedlicher Größenklassen im Kühlwasserentnahmebereich bestimmte. Die Angaben über den Jahresverlauf der Wassertemperaturen der Elbe sind Abbildung $1 \mathrm{zu}$ entnehmen.

Das Wachstum der Flundern ist von der Bestandsdichte und dem Nahrungsangebot abhängig. Ehrenbaum (1896) gibt für die Elbflunder folgende mittlere Längen am Ende des genannten Lebensjahres an: 1. Jahr $(8 \mathrm{~cm})$ 0-Gruppe, 2. Jahr $(14 \mathrm{~cm}) \mathrm{I}$-Gruppe, 3. Jahr $(19 \mathrm{~cm})$ II-Gruppe, 4. Jahr $(24 \mathrm{~cm})$ III-Gruppe. Da die Flundern aber bereits im 4. Lebensjahr vermehrt zum Meer abwandern, werden nur noch wenige Exemplare über $24 \mathrm{~cm}$ Länge in der Unterelbe angetroffen.

Während der kalten Jahreszeit dominierte die 0-Gruppe in den Kühlwasserproben, und zwar am Jahresanfang die 0-Gruppe 1977 und am Jahresende die 0-Gruppe 1978 (Abb. 3). Eine Temperaturabnahme auf Werte nahe $0^{\circ} \mathrm{C}$ führte Mitte Februar offensichtlich zu einer Abwanderung der Flundern in das wärmere, salzreichere Meerwasser der Elbmündung (Schnackenbeck, 1928). Dies bestätigte sich in einer Abnahme der Flunderzahlen von 1680 Stück/24 h auf 296 Stück/24 h Kühlwasserentnahme.

Im Verlauf des Temperaturanstieges im Frühjahr und Sommer konnte die Zuwanderung der älteren Flunderjahrgänge in der Reihenfolge I-, II- und III-Gruppe sowie einzelner älterer Tiere in den Proben beobachtet werden. Vom Sommer bis November waren die älteren Jahrgänge regelmäßig, wenn auch in wechselnder Stückzahl, in den Kühlwasserproben vertreten. Während des Fischsterbens im Mai 1978 konnte eine allgemeine Abnahme der Flunderzahlen aller Altersgruppen, nicht aber eine Dezimierung eines besonderen Jahrganges festgestellt werden (Abb. 3).

Die Einwanderung der Jungflundern des Jahrganges 1978 in die Elbmündung konnte im Kernkraftwerk Brunsbüttel von Ende Juni an registriert werden. Zu diesem Zeitpunkt variierte ihre Längenverteilung von 1-5 cm Totallänge. Bei Wassertemperaturen über $19^{\circ} \mathrm{C}(\mathrm{Abb}$. 1) spiegelte sich im August ein Wanderungsmaximum der Jungflundern einer Mindestlänge von $4 \mathrm{~cm}$ im Untersuchungsgebiet wieder. Bis zum Jahresende waren die Flundern des Jahrganges 1978 auf eine Mindestlänge von $6 \mathrm{~cm}$ herangewachsen.

Wie auch schon bei der Flunder zu beobachten war, wurde beim Stint das wechselnde Auftreten verschiedener Größengruppen von der Wassertemperatur bestimmt. Beim Schlüpfen haben die Jungstinte eine Länge von ca. 4,5-5,3 mm und wachsen nach Lillelunds (1961) Angaben bis August auf ca. $5,3 \mathrm{~cm}$ Länge heran. Als Längenmittel am jeweiligen Jahresende gibt der Autor folgende Werte für den Elbstint an: 1. Jahr (6-8 

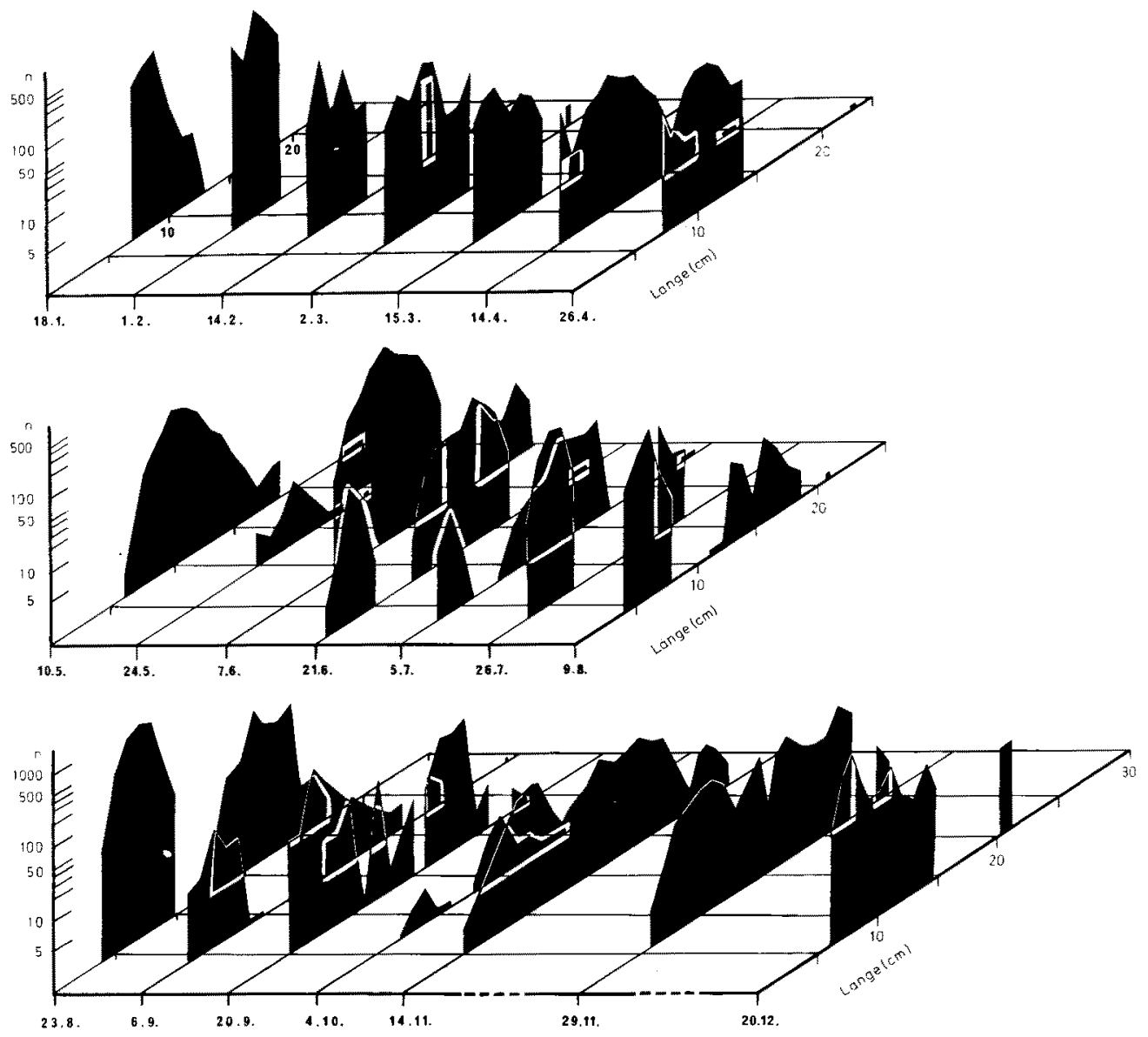

Abb. 3. Anzahl (log $\mathrm{n})$ und Längenverteilung $(\mathrm{cm})$ der Flunder (Platichthys flesus) bei einer Kühlwasserentnahme von $130000 \mathrm{~m}^{3} / \mathrm{h}$ über 24 Stunden in 21 Proben während des Jahres 1978

cm) 0-Gruppe, 2. Jahr (11,8-15,1 cm) I-Gruppe, 3. Jahr $(16,5-18,2 \mathrm{~cm}) \mathrm{II}$-Gruppe, 4. Jahr (20,3-22 cm) III-Gruppe, 5. Jahr $(23,1-24,5 \mathrm{~cm})$ IV-Gruppe. Alle diese Altersgruppen traten auch zu den verschiedenen Jahreszeiten in den Kühlwasserproben auf. Im Januar und Februar dominierte eindeutig die 0-Gruppe 1977 in den Proben (Abb. 4). In der ersten Februarwoche wurden zwar bereits einige Vorlaicher registriert, die aber bei einer Temperaturabnahme unter $1,5^{\circ} \mathrm{C}$ wieder aus den Proben verschwanden. In völliger Übereinstimmung mit den Temperaturangaben von Lillelund (1961) über Beginn 

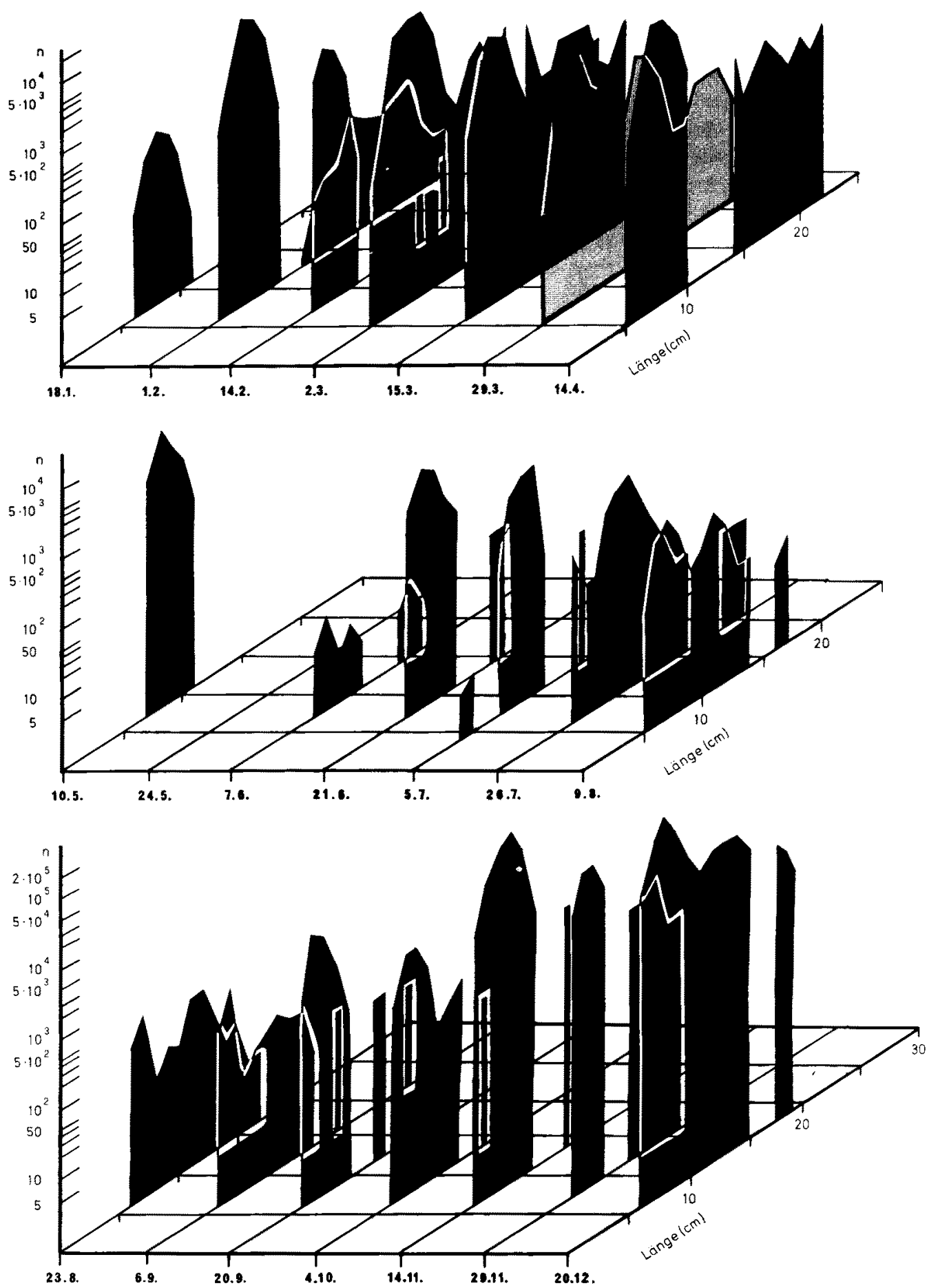

Abb. 4. Anzahl $(\log \mathrm{n})$ und Längenverteilung $(\mathrm{cm})$ des Stintes (Osmerus eperlanus) während des Jahres 1978 (vgl. Abb. 3) 
und Ende der Stintlaichzeit $\left(3,9^{\circ} \mathrm{C}-10^{\circ} \mathrm{C}\right)$ konnte die Einwanderung der Laichstinte vom 2. 3.-14. 4. 1978 in den Kühlwasserproben beobachtet werden.

Nach dem Fischsterben im Mai 1978, das zu einem völligen zeitweiligen Verschwinden der Stinte aus den Proben geführt hatte, wanderten zunächst nur einzelne Tiere der 0-, I- und II-Gruppe in den Raum Brunsbüttel ein und wurden mit dem Kühlwasser erfaßt.

Anfang Juli traten das erstemal Jungstinte des Jahrganges 1978 bei einer Wassertemperatur von $16^{\circ} \mathrm{C}$ auf. Es handelte sich um die ersten aus ihren Laichgebieten verdrifteten Jungstinte (Länge: 4-5 cm), die von den Feinrechen im Kühlwassereinlaufbauwerk erfaßt wurden (Abb. 4). In den Sommermonaten hob sich der neue Jahrgang 1978 immer deutlicher mit wachsender Stückzahl in den Proben hervor. Allerdings wurden erst größere Mengen an Jungstinten von den Feinrechen erfaßt, als sie eine Länge von $6-8 \mathrm{~cm}$ erreicht hatten. Die Zunahme entspricht der Ausdehnung des Verbreitungsgebietes der Jungstinte in Richtung zum Meer in den Sommermonaten und einer vermehrten Zuwanderung ein- und zweijähriger Stinte in die Elbmündung. Erst diese Wanderbewegungen, auch der Jungstinte aus ihren vom Sauerstoffminimum im Mai nicht betroffenen stromauf und in der Oste gelegenen Laichgebieten, brachte offenbar eine Regeneration der Elbstintpopulation nach dem Fischsterben. Dies ist an der Größenzusammensetzung und der Zunahme der Stinte zu erkennen (Abb. 4). Der enorme Anstieg der Stintzahlen der 0-, I- und II-Gruppe im November und Dezember bis auf 647902 Stück/24 h spiegelte die winterliche Ansammlung der Stintpopulation aus dem Fluß und von See im Raum Brunsbüttel-Cuxhaven wieder.

Das Auftreten der verschiedenen Altersgruppen des Aales in den Kühlwasserproben im Jahre 1978 paßte sich völlig in das aus der Literatur bekannte Bild über temperaturabhängige Verhaltensweisen dieser Art ein (Mann, 1963; Aker \& Koops, 1970; Tesch, 1967, 1977). So wirkte der Temperaturanstieg auf 8-10 ${ }^{\circ} \mathrm{C}$ Anfang April (Abb. 1) aktivitätsauslösend auf die Wandenung der Jungaale, die zunehmenden Nahrungswanderungen der Gelbaale und die im Vorherbst abgebrochenen, nunmehr fortgesetzten Wanderungen der Blankaal- $q$ ( $\mathrm{Abb}$. 5). Außerdem stimmten die Längenhäufigkeiten der Aale in den Kühlwasserproben mit Ausnahme der kleineren Glasaale mit den Längenangaben für die im Gewässer natürlich vorkommenden Altersgruppen überein, und zwar für Jungaale bis $30 \mathrm{~cm}$, für Gelbaale $>30 \mathrm{~cm}$, für Blankaal- ơ đo von $35-45 \mathrm{~cm}$ und für Blankaal- $q q$ über $50 \mathrm{~cm}$ Länge (Tesch, 1977) (Abb. 5).

Nachdem während des Sauerstoffminimums im Mai 1978 ein erheblich größerer Gelbaalanteil gegenüber dem Jungaalanteil als in den Proben zuvor mit dem Kühlwasser angesogen wurde, sanken in den folgenden Proben bis zum Monat August die Gelbaalanteile gegenüber den Jungaalanteilen von $30 \%$ bis auf Minimalwerte von $7,3 \%$, wie aus dem Diagramm über die Längenverteilung des Aales zu entnehmen ist. Es liegt also die Schlußfolgerung nahe, daß bei dem Fischsterben im Mai 1978 vornehmlich der Gelbaalbestand im Bereich Brunsbüttel betroffen war. Normalerweise wäre bei sommerlichen Wassertemperaturen nach Thurow (1959) ein Anstieg der Gelbaalzahlen zu erwarten gewesen. Die relativ hohe Toleranz adulter Aale gegenüber einer Sauerstoffabnahme (vgl. Tesch, 1977) und die hohe Standorttreue dieser Aalform (Mann, 1965) hatte offenbar eine rechtzeitige Abwanderung verhindert. Die Jungaale sind dagegen vermutlich aufgrund ihrer größeren Sauerstoffempfindlichkeit und einer vermehrten Wanderbereitschaft zu einem größeren Teil in der Lage gewesen, den betroffenen 

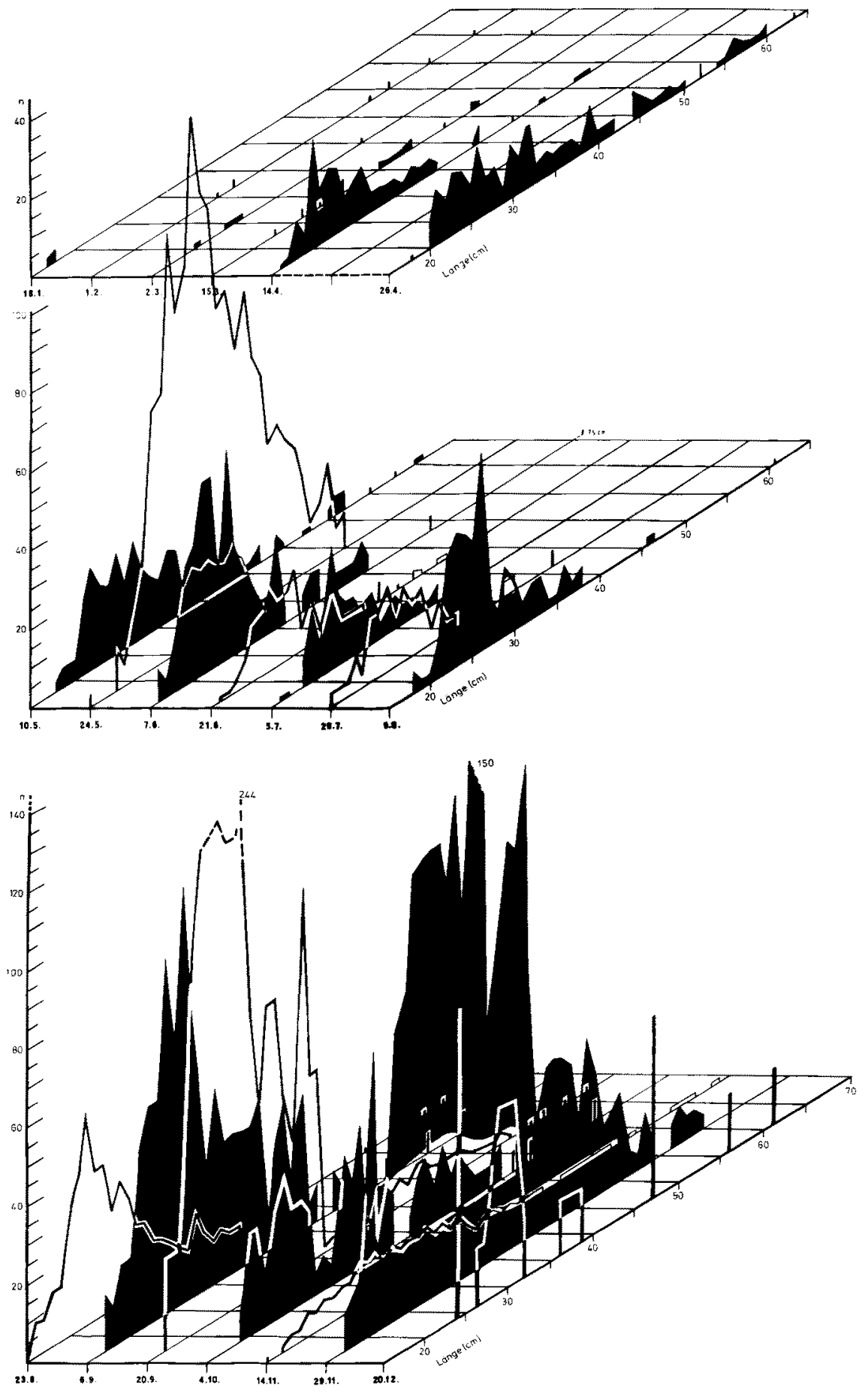

Abb. 5. Anzahl (n) und Längenverteilung des Aales (Anguilla anguilla) während des Jahres 1978 (vgl. Abb. 3) 
Flußabschnitt rechtzeitig zu verlassen. Dies wird besonders in der Probe vom 7. 6. 1978 deutlich. Zu diesem Zeitpunkt stabilisierten sich die Sauerstoffverhältnisse durch eine hohe Oberwasserzufuhr, die gleichzeitig die Zuwanderung einer vergleichsweise hohen Zahl an Jungaalen, wahrscheinlich aus dem oberen Elbabschnitt, mit sich brachte (Abb. 5).

Anfang September kündigte sich der Beginn der herbstlichen Blankaalwanderung an, die sowohl aus dem Anstieg der Gesamtzahlen (Abb. 5) als auch an der veränderten Längenhäufigkeit der Aale zu erkennen ist (Abb, 4). Dabei nahm bei sinkenden Temperaturen auf $16^{\circ} \mathrm{C}$ der Anteile der Aale mit Längen zwischen 35 und $45 \mathrm{~cm}$ erheblich zu (Abb. 4); Aale mit einer Länge von über $50 \mathrm{~cm}$ wurden zu diesem Zeitpunkt nur vereinzelt angetroffen. Wie der Verlauf der Längenhäufigkeit in den folgenden Proben zeigte, wuchs der Anteil größerer Blankaale von Anfang Oktober bis Ende November erheblich an. Die im küstennahen Bereich lebenden kleineren Blankaale erreichten offenbar den Bereich Brunsbüttel eher, als die aus dem Oberlauf der Elbe und ihren Nebengewässern abwandernden großen Blankaale. Bei Wassertemperaturen unter $9^{\circ} \mathrm{C}$ deutete sich in den Kühlwasserproben ein Wanderungsmaximum der Blankaale beiderlei Geschlechts und eine erhöhte Wanderaktivität der Jung- und Gelbaale in die Winterquartiere im Süßwasser an (Aker \& Koops, 1973). Im Dezember sanken die Wassertemperaturen unter $3^{\circ} \mathrm{C}$ und der Kälteeinbruch beendete die Wanderungsaktivität der verschiedenen Aalformen (Abb. 4). Im übrigen fiel bei der Beobachtung der Blankaale auf, das sich die Erkrankungen Rotseuche und Blumenkohlerkrankung ausschließlich auf Aale einer Länge bis $45 \mathrm{~cm}$, den stärker schadstoffbelasteten Unterelberaum bewohnende Aalbestände, beschränkten. Große Aale der oberen Elbe und deren Nebengewässer zeigten keine dieser Krankheitssymptome. Insgesamt wurden 1342 Aale daraufhin untersucht (Köhler, unveröffentlicht).

\section{Weitere Fischarten}

Die Fischarten Stichling (Gasterosteus aculeatus L.), Finte (Alosa fallax [Lacepede]) und Neunauge (Lampetra fluviatilis L.) wandern nur zum Laichen in die Elbmündung ein. Für ihr periodisches Auftreten in den Kühlwasserproben konnte eine Korrelation zum jahreszeitlichen Verlauf der Wassertemperatur gefunden werden (Abb. 1). Über die Grundel (Pomatoschistus microps L.) kann nach bisherigen Untersuchungen nur festgestellt werden, daß sie im Spätsommer, wahrscheinlich zur Nahrungsaufnahme, ins Süßwasser wandert (Totzke, mündliche Mitteilung).

In der Elbe und in der Nordsee ist der dreistachelige Stichling (Gasterosteus aculeatus L.) als marine Mischpopulation verschiedener Beschilderungstypen (trachurus, semiarmatus, leiurus) verbreitet (Münzing, 1959, 1962, 1963). Die Angaben Münzings (1959), der Stichling halte sich im Herbst und Winter nicht in der Unterelbe auf, konnte nicht bestätigt werden. In den Herbst- und Winterproben 1977/78 und 1978/79 wurden einige hundert bis einige tausend Stichlinge gezählt (Abb. 6). Anfang Februar begann ähnlich früh, wie auch Baggermann (1957) für die niederländische Küste berichtet hat, die Frühjahrswanderung der Stichlinge in die Elbe. Bei steigenden Temperaturen von März bis Anfang Mai auf ca. $12^{\circ}-14^{\circ} \mathrm{C}$ (Abb. 1) setzten sich in Übereinstimmung mit den Verhaltensexperimenten von Baggermann (1957) und Angaben von Wootton (1976) die massiven Wanderungen des Stichlings fort. Dies ist der 
Abbildung 6, erkennbar an den hohen Stückzahlen bis 50614 Stück/24 Stunden Kühlwasserentnahme, zu entnehmen. Ende Mai und im Juni wurden in geringen Mengen meerwärts wandernde abgelaichte Fische beobachtet. Anfang Juli konnte bei Wassertemperaturen von $16-17^{\circ} \mathrm{C}$ mit $5084 \mathrm{Stück} / 24 \mathrm{~h}$ ein sommerliches Stichlingsmaximum in den Proben verzeichnet werden. Die Probe setzte sich aus Jungfischen einer Länge von $2-5 \mathrm{~cm}$ und adulten Stichlingen zusammen. Die Jungfische entwickeln 2 Monate nach dem Schlüpfen in den tideabhängigen Gräben der Unterelbe eine Präferenz für höhere Salinitäten (Baggermann, 1957); sie sind dann bei einer Größe von ca. $2 \mathrm{~cm}$ in der Elbmündung anzutreffen (Münzing, 1959). Bei sinkenden Temperaturen (auf $7^{\circ} \mathrm{C}$ im November und $2{ }^{\circ} \mathrm{C}$ im Dezember) wurden $300-400$ Stichlinge $/ 24 \mathrm{~h}$ in den Proben gesammelt (Abb. 6).

Nach dem Ausbleiben des echten Maifisches (Bauch, 1958) kommt nur noch die Finte (Alosa fallax [Lacepede]) in der Unterelbe vor. Die Finte hält sich nur kurz während der Fortpflanzungszeit in der Elbe auf (Schnackenbeck, 1928) und laicht im Gebiet von der Este-Mündung bis Brunsbüttel von Ende April bis Anfang Juni. Die Hauptwanderzeit liegt im Mai.

Ende März und Mitte April traten jeweils nur eine Finte in den Proben auf. Anfang Mai jedoch wurde bei Temperaturen von $11-12^{\circ} \mathrm{C}$ auch in den Kühlwasserproben ein gehäuftes Auftreten dieser Fischart verzeichnet (Abb. 6). Es konnten fast ausschließlich adulte, laichreife Tiere (Länge: $29-36 \mathrm{~cm}$ ) beobachtet werden. Ende Juni bis Anfang August wurden vereinzelt abwandernde adulte Finten angesogen. Von Ende August an traten Jungfinten bis zum Jahresende in den Proben auf; die Jungfische wiesen von Ende August bis Ende September eine Länge von $3-5 \mathrm{~cm}$ auf (16-25 Stück/24 h). Im Oktober und November wanderten die Jungfinten bei fallenden Temperaturen in

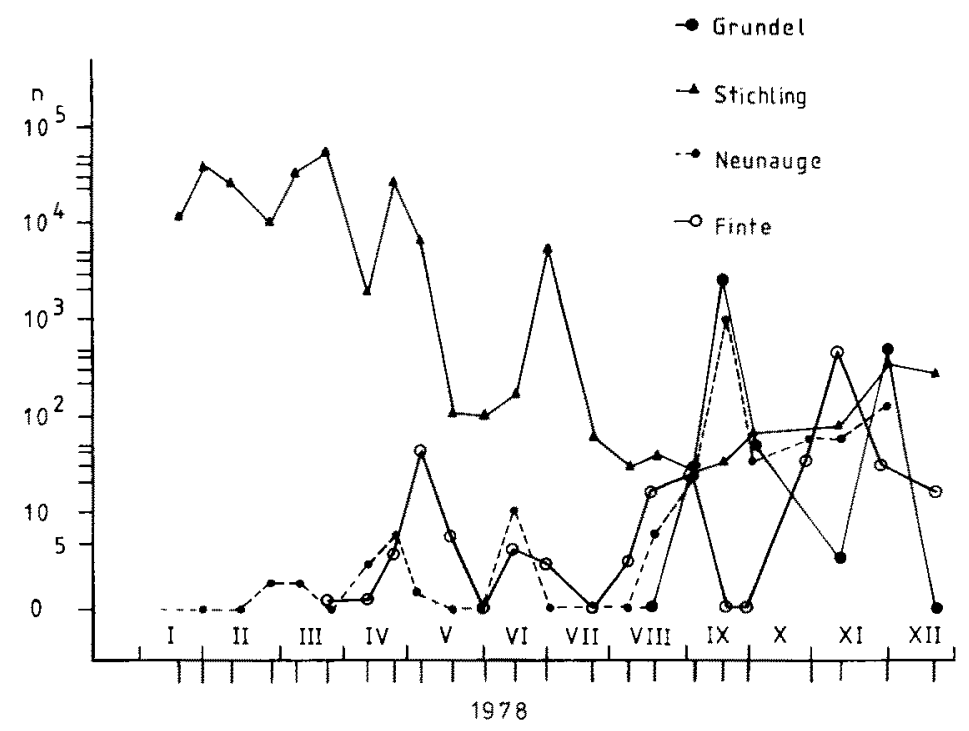

Abb, 6. Verteilung von Grundel (Pomatoschistus microps), Neunauge (Lampetra fluviatilis), Stichling (Gasterosteus aculeatus) und Finte (Alosa fallax) in 23 Kühlwasserproben uber eine Dauer von jeweils 14 Stunden bei einem Durchfluß von $130000 \mathrm{~m}^{3} / \mathrm{h}$ 
wachsender Zahl nach Brunsbüttel, wie in dem Jahresmaximum von 424 Stück $/ 24 \mathrm{~h}$ zu erkennen ist. Sie waren nunmehr auf $6-8 \mathrm{~cm}$ Totallänge herangewachsen. Dabei könnte der erhöhte herbstliche Oberwasserabfluß einen Einfluß auf die Abwanderung der Jungfinten zusätzlich ausgeübt haben, da jeweils nachfolgend auf eine Oberwassererhöhung ein Anstieg der Jungfinten zu verzeichnen war (Abb. 1 und 6). Bei sinkenden Temperaturen auf $2-4{ }^{\circ} \mathrm{C}$ bei Brunsbüttel nahmen die Jungfinten Ende November und im Dezember in den Proben ab.

Sowohl das Meerneunauge (Petromyzon marinus L.) als auch das Flußneunauge (Lampetra fluviatilis L.) kamen um die Jahrhundertwende massenhaft in der Elbe vor (Bauch, 1958). Als Folge der zunehmenden Elbverschmutzung gilt der Fang eines Meerneunauges (60-75 cm Länge) heute als Rarität; das kleinere Flußneunauge (32-34 $\mathrm{cm}$ Länge) kommt noch, wenn auch mit stark abnehmender Tendenz, in der Unterelbe vor (Wilkens \& Köhler, 1977).

Von Anfang März bis Anfang Mai wurden bei Wassertemperaturen über $7^{\circ} \mathrm{C}$ ausschließlich adulte Tiere einer Länge von $29-36 \mathrm{~cm}$ in nur geringer Zahl, wie auch von Iman et al. (1958) beobachtet wurde, in den Proben vermerkt (Abb. 6). Danach hielten sich in den Sommermonaten bis August keine erwachsenen Flußneunaugen im Gebiet Brunsbüttel auf. Dagegen wurden junge Neunaugen (18-19 cm Länge) am 21. 6 . 78 angesogen. Sie befanden sich vermutlich auf ihrer Abwanderung ins Meer, nachdem sie 3-5 Jahre in ihren Laichgewässern herangewachsen waren (Iman et al., 1958).

Nach Tesch (1967) finden die herbstlichen Laichwanderungen der Flußneunaugen flußaufwärts bei Nacht ca. 4 Tage nach Voll- oder Neumond statt. Diese Vermutung bestätigte sich in der vorliegenden Untersuchung in eindeutiger Weise. Allerdings zeigte sich die Lunarabhängigkeit nicht nur für die herbstliche Laichwanderung, sondern auch für die Wanderaktivitäten während des übrigen Jahres. Am 23: 4. 1978 war Vollmond, und in der Probe am 26. 4. konnte ein leichter Neunaugenanstieg bemerkt werden; am 21. 6. wurde eine Abwanderung von jungen Neunaugen registriert (Abb. 6), am 20. 6. war Vollmond.

Die herbstliche Laichwanderung begann sich vom 23. 8. an in den Proben anzudeuten: 5 Tage nach Vollmond (18. 8. 1978) wurden die ersten erwachsenen Neunaugen bei Temperaturen von $19,8^{\circ} \mathrm{C}$ in den Proben registriert. Die Spitzenzahlen an Neunaugen (938 Stück/24 h) brachte jedoch die Probe vom 20. 9. 1978 bei Wassertemperaturen von $14,3^{\circ} \mathrm{C}, 4$ Tage nach Vollmond (am 16.9). Wahrscheinlich müssen nach vorliegenden Beobachtungen mehrere Faktoren zusammentreffen, um ein Aktivitätsmaximum der Laichwanderungen von Lampetra fluviatilis auszulösen. Diese Faktoren sind Mindesttemperaturen unter $15^{\circ} \mathrm{C}$ in Kombination mit einer bestimmten Mondphase (einige Tage nach Vollmond).

In den Proben bei Brunsbüttel trat die Grundel (Pomatoschistus microps L.) nur im Herbst auf. Diese Grundelart scheint nach Totzke (mündliche Mitteilung) als Jungfisch eine Präferenz für niedrige Salzgehalte zu haben, so daß eine Einwanderung in den Süßwasserbereich der Elbe selbst bis Hamburg möglich ist.

Im Kernkraftwerk Brunsbüttel wurden im Jahr 1978 erst Anfang September Grundeln in den Proben gefunden. Diese wiesen eine Länge von $4 \mathrm{~cm}$ und mehr auf, so daß man annehmen kann, daß kleinere, eher einwandernde Exemplare vom Feinrechen nicht erfaßt wurden. Ein Maximum der Grundelzahlen wurde am 20. 9. 1978 mit 2106/ $24 \mathrm{~h}$ festgestellt. Bis Mitte November wurden danach nur noch wenige Tiere angesogen 
(Abb. 6). Ein weiteres Maximum konnte am 29. 11. verzeichnet werden. Zu Beginn der Untersuchungen im Herbst 1977 konnte am 30. 11. ein ähnlicher Anstieg beobachtet werden. Dabei handelte es sich jeweils wahrscheinlich um Grundeln, die nach Beendigung ihres Süßwasseraufenthaltes bei sinkenden Temperaturen ins wärmere tiefere Seewasser zurückkehrten (Totzke, mündliche Mitteilung). Im Dezember wurden bei Wassertemperaturen unter $3^{\circ} \mathrm{C}$ keine Grundeln mehr registriert.

Der Kaulbarsch (Gymnocephalus cernua L.) bewohnt den Süß- und Brackwasserbereich der Flüsse. Die von Elbfischern berichtete Empfindlichkeit des Kaulbarsches auf Salzgehaltsschwankungen zeigte sich auch für den Untersuchungszeitraum von 1978. In nennenswerten Mengen wurde diese Art nur bei einer S a li n i tä $t$ von ca. $2,5-3,5 \%$ in den Proben beobachtet. Auf Abbildung 7 wird dies in den Zeitabschnitten Mitte-Ende Februar und in den Proben vom 21.6, 26.7. 23. 8., 20.9. und 29. 11. deutlich. Es muB allerdings ausdrücklich betont werden, daß es sich bei den Salinitätsangaben um Schätzwerte handelt, die nach Angaben Luchts (1965) über die Verschiebung der Brackwasserregion durch den Oberwasserabfluß unter Berücksichtigung der "flushing time" ermittelt wurden (Abb. 1). Verwendbare Meßdaten über die Salinität im Raum Brunsbüttel liegen für das Jahr 1978 nicht vor.

Das Eindringen der Heringsartigen Sprott und Hering, anderer mariner Fischarten und der Garnelenarten Crangon crangon und Palaemon longirostris wurde, wie die Untersuchung zeigte, vorwiegend vom wechselnden Salzgehalt bei Brunsbüttel bestimmt. Hering (Clupea harengus L.) und Sprott (Sprattus sprattus L.) verschwanden aus den Proben, sofern die Oberwasserzufuhr $1000 \mathrm{~m}^{3} / \mathrm{s}$ überstieg, gefolgt von einer Salzgehaltserniedrigung auf ca. 1,2\%. Im März/April und Mai/Juni wurden diese Abhängigkeiten in den Proben deutlich. Bei Niedrigwasserabflüssen erreichten die Salzgehalte Werte bis $6,8 \%$ bei Brunsbüttel; Salzgehalte über $2 \%$ erlaubten den Herings- und Sprottschwärmen die Einwanderung in die Elbmündung. Nach Tiews (1960) handelt es sich hauptsächlich um einjährige Heringe (Spitzen).

Die typische Brackwassergarnele Palaemon longirostris L. war stets in größerer Zahl als die Nordseegarnele Crangon crangon Fabr. in den Kühlwasserproben vertreten. Im Januar und März kamen nur wenige Garnelen vor, erst ab Ende April wuchs die Anzahl auf $1200 / 24 \mathrm{~h}$ bei nachlassender Süßwasserzufuhr und steigenden Wassertemperaturen (Abb. 7). Von Juli an bis einschließlich Oktober steigerte sich der Garnelenanteil auf 50963 Stück $/ 24 \mathrm{~h}$ bei durchschnittlichen Abflüssen von $400 \mathrm{~m}^{3} / \mathrm{s}$ und einer Salinität von schätzungsweise 3,5-6,8\%. Dieses in Brunsbüttel zu beobachtende massive Garnelenauftreten stimmt mit den Jahresfangmaxima der Deutschen Garnelenfischerei, deren Ergebnisse Tiews (1971) über einen Zeitraum von 6 Jahren beschrieben hat, überein. Seefischarten kamen ebenfalls in der Regel vereinzelt nur in Zeiten erhöhter Salinität im Untersuchungsgebiet vor (Abb. 7). In erster Linie handelte es sich dabei um kleine Seezungen (Solea solea L.) mit einer Länge von 9-11 cm. Daneben kamen vereinzelt andere Plattfische vor, wie Steinbutt (Psetta maxima L.), Flügelbutt (Lepidorhombus whiff-iagonis [Walb.]) und Scharbe (Hippoglossoides platessoides [Fabr.]). Auch der Franzosendorsch (Trisopterus luscus [L.]) und besonders im Juni und Juli der Sandaal (Ammodytes tobianus [L.]) traten im Kühlwasser auf.

Weißfischarten (Cyprinidae) kamen während des Untersuchungszeitraumes in Brunsbüttel stets nur in Einzelexemplaren vor. Es handelte sich um einzelne Brassen (Abramis brama L.), Güster (Blicca björkna L.), Zope (Abramis ballerus L.) und Rotfeder 


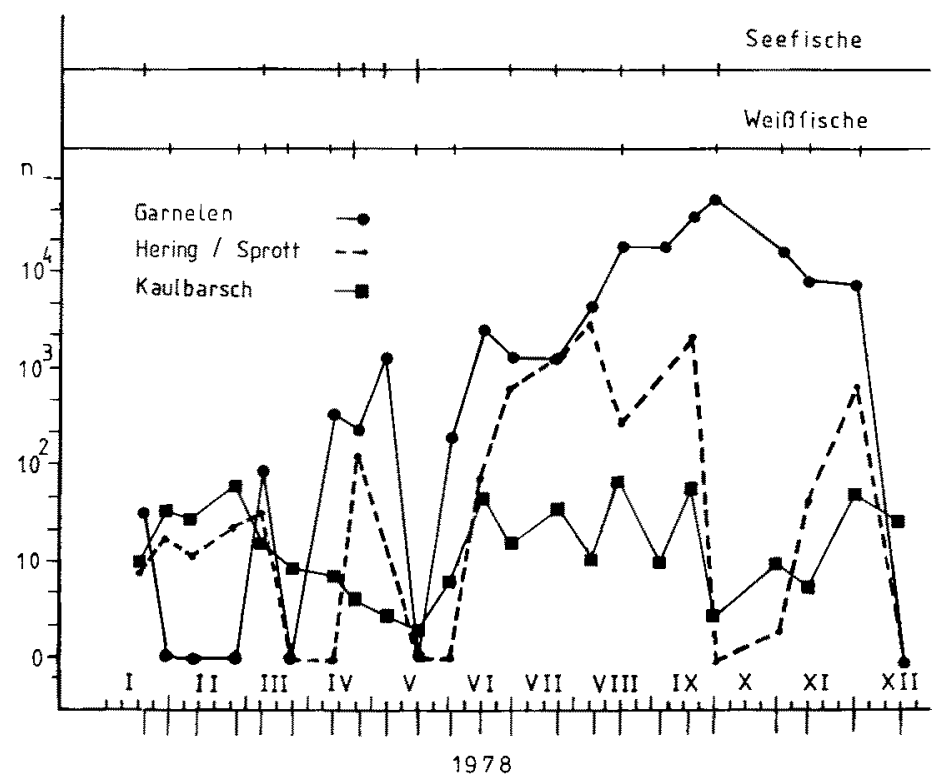

Abb. 7. Verteilung von Heringsartigen (Clupea harengus und Sprattus sprattus), Garnelenarten (Palaemon longirostris und Crangon crangon), Kaulbarsch (Gymnocephalus cernua) und verschiedenen Weißfisch- und Seefischarten in 23 Kühlwasserproben über eine Dauer von jeweils 24 Stunden bei einem Durchfluß von $130000 \mathrm{~m}^{3} / \mathrm{h}$ im Kernkraftwerk Brunsbüttel

(Scardinius erythrophthalmus L.). Des öfteren waren auch Zander (Stizostedion lucioperca [L.]) gemeinsam mit den Cypriniden anzutreffen.

\section{Bilanz der Fischverluste}

Im Untersuchungsjahr 1978 wurden nur 72,6\% der geplanten Kühlwassermenge von $1205848000 \mathrm{~m}^{3}$ auf Grund des zeitweiligen Stillstandes des Kernkraftwerkes Brunsbüttel der Elbe entnommen. Die Mehrzahl der Probenahmen fand bei einer Kühlwasserentnahme von $120000 \mathrm{~m}^{3} / \mathrm{h}\left(130000 \mathrm{~m}^{3} / \mathrm{h}\right.$ geplant $)$ statt; wurden bei einzelnen Probenahmen geringere Kühlwassermengen gefahren, sind die angesogenen Fischmengen auf die "Normal" entnahme hochgerechnet worden. Für die Fischarten, deren Auftretenshäufigkeit während des Jahres stark in den Proben schwankte, wurden für die Interpolation der vernichteten Gesamtmengen auf das Jahr die Phasen der Häufigkeitsmaxima und -minima der Berechnung zugrunde gelegt (Tab. 1). Bei einem relativ gleichmäßigen Auftreten, zumindest während eines Quartales, wurde die Berechnung der Fischmengen nach Quartalen vorgenommen (Tab. 2).

Wollhandkrabben (Erioreir sinensis Milne Edwards) gingen auf Grund unregelmäBiger Auszählungen in den Proben nicht in die Auswertung mit ein. Die Berechnungen haben ergeben, daß während eines Jahres bei voller Kühlwasserentnahme $190659 \mathrm{~kg}$ Fische und Krebstiere von den Rechen aus dem Kühlwasser ferngehalten werden. Dies entspricht einer Individuenzahl von ca. 27500000 Stück. Das Vorkommen verschiede- 


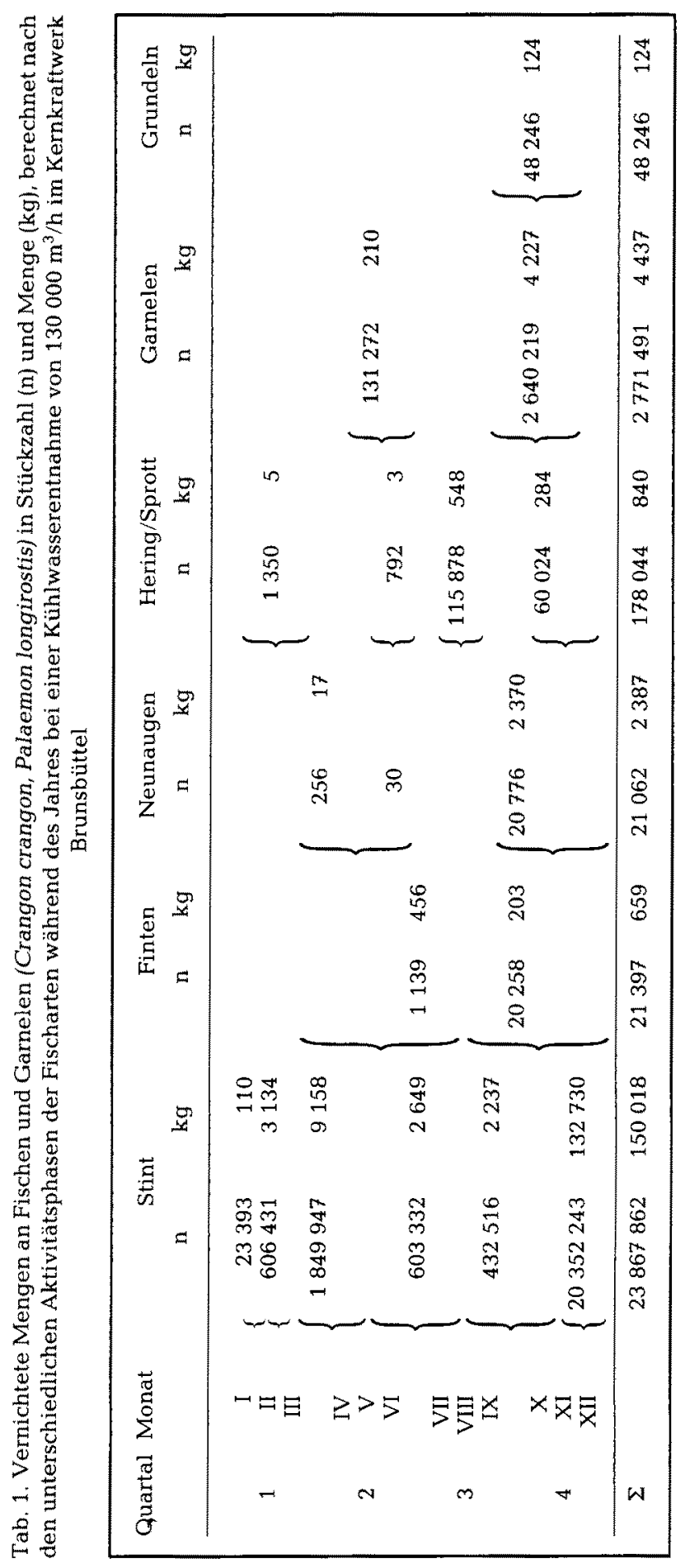


Tab. 2. Vernichtete Fischmengen in Stückzahl (n) und Menge (kg) pro Quartal bei einer Kühlwasserentnahme von $130000 \mathrm{~m}^{3} / \mathrm{h}$ im Kernkraftwerk Brunsbütel

\begin{tabular}{|crrrrrrrrrrr}
\hline \multirow{2}{*}{ Quartal } & \multicolumn{2}{c}{ Aal } & \multicolumn{4}{c}{ Flunder } & \multicolumn{2}{c}{ Stichling } & \multicolumn{2}{c}{ Kaulbarsch } \\
& $\mathrm{n}$ & $\mathrm{kg}$ & \multicolumn{2}{c}{$\mathrm{n}$} & $\mathrm{kg}$ & $\mathrm{n}$ & $\mathrm{kg}$ & $\mathrm{n}$ & $\mathrm{kg}$ \\
\hline 1 & 3672 & 179 & 60115 & 570 & 2589495 & 7400 & 2302 & 238 \\
2 & 47058 & 1929 & 58910 & 911 & 510783 & 1650 & 1052 & 107 \\
3 & 60845 & 2594 & 80398 & 1032 & 80947 & 92 & 3065 & 312 \\
4 & 76713 & 6368 & 37034 & 7796 & 16904 & 19 & 2090 & 212 \\
\hline$\Sigma$ & 188289 & 11070 & 355482 & 10309 & 3198129 & 9160 & 8509 & 1526 \\
\hline
\end{tabular}

ner Größenklassen im Laufe des Jahres spiegelte sich in der Relation von der Stückzahl der Fische zu ihrem Gewicht wieder. Besonders deutlich wurde dies bei Finte und Flunder (Tab. 1 und 2).

Bis auf Jungstinte, Jungfinten, Heringe und Sprott waren die mit dem Kühlwasser angesogenen Fische und Garnelen noch am Leben. Allerdings traten zum Teil erhebliche Verletzungen durch die Rechen wie Quetschungen (Flundern, Rundfische) oder Durchtrennungen (Aale) auf. Nicht von den Rechen zurückgehalten wurden Flundern ( $<1 \mathrm{~cm}$ Länge), Grundeln ( $<4 \mathrm{~cm}$ Länge) und Stichlinge $(<2 \mathrm{~cm}$ Länge). Die Mehrzahl der Stinte $(<5 \mathrm{~cm}$ Länge) sowie die Glasaale von weniger als $14 \mathrm{~cm}$ Länge wurden auf Grund ihrer spezifischen Körperform nicht abgefangen.

Larven und Fischeier, neben anderen Zooplanktern und Phytoplanktern passieren ebenfalls das Rektorkühlsystem und erfahren eine Temperaturerhöhung um mindestens $10^{\circ} \mathrm{C}$ (Werksbericht Kernkraftwerk Brunsbüttel, 1978). Eine Bilanzierung der PlanktonBiomasse, die nach bisherigen Untersuchungsergebnissen durch die Erwärmung geschädigt oder vernichtet wird (Benda, 1974; Kellogg, 1977; Schubel, 1977; Hettler, 1978; Gaudy, 1979; Donze, 1979; Capuzzo, 1980), wurde nicht vorgenommen. Für das Elbe-Ästuar liegen keine neueren produktionsbiologischen Untersuchungen vor, auf deren Basis eine derartige umfassende Bilanzierung hätte vorgenommen werden können. Abgesehen von der ökologischen Beeinträchtigung der Elbmündung, auch durch die Zerstörung von Fischbrut und Nahrungsorganismen (Jungfische, Larven, Laich, Plankton) liegt eine direkte, nicht unerhebliche Schädigung der Fischerei vor, insbesondere deshalb, weil die zum größten Teil lebenden Fische nicht in die Elbe zurückgeleitet, sondern als Abfall der Müllbeseitigung zugeführt werden.

\section{DISKUSSION}

Während des Jahres 1978 war mit erheblichen jahreszeitlichen Schwankungen das gesamte derzeitige Fischartenspektrum der Elbe in allen vorkommenden Größenklassen im Kühlwasser des Kernkraftwerkes Brunsbüttel vertreten. Das Reaktorkühlsystem passiert haben nur diejenigen Fische einer bereits erwähnten Mindestlänge und planktische Organismen, die von den Rechen nicht erfaßt wurden. In den Proben zeigte sich, wie die Wassertemperatur im Jahresverlauf Beginn und Ende der Laichzeiten, das Auftreten der Jahrgänge 1978 und die Wanderungsaktivitäten der verschiedenen 
Fischaltersgruppen bestimmte. In Abhängigkeit vom Oberwasserzufluß aus der oberen Elbe schwankten die Sauerstoffgehalte der Unterelbe und beeinflußten so das quantitative Vorkommen der einzelnen Fischarten. Dies spiegelte sich in den Kühlwasserproben wieder.

Mit dem Kühlwasser wurden nicht, wie zunächst anzunehmen war, selektiv junge, kranke oder alte Fische angesogen. Die Beobachtungen legen nahe, daß das in den Kühlwasserproben analysierte Arten- und Größenspektrum ein repräsenatives Bild für das von abiotischen und anthropogenen Faktoren beeinflußte Fisch- und Garnelenvorkommen im unteren Elbe-Ästuar während eines Jahres vermittelt.

Die Fischscheuchanlage zeigte keinerlei Effekt. Die dadurch entstehende jährliche Schädigung der Elbfischerei läßt sich an Hand einiger Zahlen der Gesamtkutteranlandungen am Seefischmarkt Cuxhaven verdeutlichen. Im Untersuchungsjahr 1978 wurden in Cuxhaven $528271 \mathrm{~kg}$ Fisch von den in der Elbmündung fischenden Kuttern angelandet, davon bestanden $54 \%$ aus Seefischen und Beifang; 1979 nahmen die Anlandungen auf $315247 \mathrm{~kg}$ ab, $48 \%$ davon waren Seefische und Beifang *. Bei Vollbetrieb der Kühlwasseranlage des Kernkraftwerkes Brunsbüttel von $130000 \mathrm{~m}^{3} / \mathrm{h}$ werden nach den vorliegenden Untersuchungsergebnissen jährlich mindestens $190000 \mathrm{~kg}$ Fische und Garnelen angesogen. Die mit dem Kühlwasser der Elbe entnommene Fischmenge ist also den Elbfischkutteranlandungen vergleichbar. Seit den im Jahre 1978 durchgeführten Untersuchungen sind keine Umbauten im Kühlwassereinlaufbauwerk zwecks Rückführung der angesogenen Fische oder aber an der Fischscheuchanlage zur Verringerung des Fischanfalles vorgenommen worden. Damit ist der Sachverhalt einer nicht unerheblichen fischereilichen Schädigung durch die Kühlwasserentnahme (seit dem 22. 6. 1976) bei einer vorauszusetzenden vollen Pumpenleistung gegeben.

Da für das Elbe-Ästuar keine aktuellen, umfassenden produktionsbiologischen Untersuchungen für den Zeitraum der wachsenden industriellen Besiedlung der Elbrandzonen vorliegen, konnte eine Abschätzung einer Schädigung der Planktonbiomasse und der Fischbrut durch die Kühlwasserentnahme nicht vorgenommen werden.

Abgesehen von möglichen ökologischen Schädigungen durch eine Kühlwasserentnahme spiegelten sich in der qualitativen und quantitativen Zusammensetzung der Fisch- und Garnelenproben Beeinträchtigungen des Sauerstoffgehaltes im Unterelberaum wieder. Eindeutige anthropogene Störungen des Okosystems ließen sich an den Fischarten aufzeigen, welche sich über eine längere Zeitspanne im Elbe-Ästuar aufhielten. Die Analyse des Flunderauftretens in den Proben unter Beachtung der Sauerstoffverhältnisse im ehemaligen flußaufwärts gelegenen und im heutigen Hauptverbreitungsgebiet bei Brunsbüttel bis Cuxhaven deutete auf sauerstoffabhängige Wanderungen zwischen den beiden Gebieten hin. Bestätigt wurde diese Verhaltensweise durch die Überprüfung der Fangerträge bei Pagensand (Stromkilometer 642), die bei schlechten Sauerstoffbedingungen sofort auf Null sanken, bei hohem Oberwasser und verbesserten Sauerstoffgehalten aber wieder zunahmen (Köhler, unveröffentlicht).

Die Untersuchungsergebnisse ergaben, daß sich die Stint-Laichzeit im Jahre 1978 auf mindestens 44 Tage gegenüber 26,8 Tagen Ende der fünfziger Jahre (Lillelund, 1961) verlängert hatte. Diese Tendenz wurde schon von Brinkmeyer (1978) festgestellt, der eine Stintlaichzeit von 37 Tagen ermittelt hat. Weiterhin fiel auf, daß die früher stets

\footnotetext{
* Angaben der Statistischen Abteilung des Seefischmarktes Cuxhaven.
} 
zu beobachtende rapide Abnahme der Fangerträge im Mündungsgebiet, welche sich in den Proben im Kühlwasser des KKW Brunsbüttel widergespiegelt haben müßte, ausblieb. Nur bei hohen Oberwasserabflüssen und einer entsprechenden Verdünnung der "Schmutzfracht" der Unterelbe wanderten die Stinte vermehrt flußaufwärts in die Richtung ihrer ehemaligen Laichgebiete oberhalb Hamburgs. Während der übrigen Zeit stauten sich die Stintschwärme offenbar im Raum Brunsbüttel, wie an den extrem hohen Stintzahlen in den Frühjahrsproben zu beobachten war. Der intensive flußaufwärtsgerichtete Wandertrieb mancher Fischarten, sofern die Sauerstoffbedingungen dies zulassen, ist nach Hynes (1977) ein entscheidender Faktor zur Wiederbesiedlung zuvor verlassener Flußabschnitte.

Die Beeinträchtigung des Fischlebensraumes im unteren Elbe-Ästuar zeigte sich besonders kraß im Mai 1978. Ein außergewöhnliches Sauerstoffminimum führte zu dem größten Fischsterben seit 30 Jahren im Raum Brokdorf-Neufeld (Stromkilometer 685-725) vom 19.5.-2. 6. 1978. Eine Verschiebung der organisch hochbelasteten Zone zwischen Hamburg und Glückstadt durch eine hohe Oberwasserzufuhr aus der oberen Elbe kann nicht die Ursache dieses Sauerstoffminimums im Mai gewesen sein. Die im Mai bei Brunsbüttel gemessenen Stickstoffgehalte wurden im Laufe des Sommers 1978 noch weit überschritten, ohne daß bei Temperaturen von $17-20^{\circ} \mathrm{C}$, also mehrfach erhöhter Nitrifikation (Rheinheimer, 1971), ein annähernd vergleichbares Sauerstoffminimum registriert werden konnte. Ein sich flußabwärts aus dem Raum Wedel nach Brunsbüttel verlagerndes "Sauerstoffloch" hätte sich bei niedrigen Oberwasserabflüssen ca. 2 Wochen vorher in den Meßwerten der Stromstrecke von Hamburg bis Glückstadt ankündigen müssen (vgl. $\mathrm{O}_{2}$-Gehalte bei Wedel, Abb. 1). Das Sauerstoffminimum wurde nur in den Einzelmessungen bei Brunsbüttel und unterhalb festgestellt (ARGE Elbe, 1978; Abb. 1). Als ein eindeutiges Indiz für unnatürliche sauerstoffzehrende Prozesse in der Elbmündung selbst ist die Tatsache anzusehen, daß die täglichen Sauerstoffmessungen bei Cuxhaven über einen Zeitraum von 14 Tagen für dieses Gebiet konstant außerordentlich niedrige $\mathrm{O}_{2}$-Gehalte von 4,3-6 mg/l aufwiesen (ARGE Elbe, 1978). Handelte es sich bei dem Sauerstoffdefizit um ein elbabwärts verlagertes "Sauerstoffloch", müßten extrem schwankende Sauerstoffwerte auf Grund des Eindringens sauerstoffreicheren Meerwassers mit der Tide im Raum Cuxhaven gemessen worden sein (Maier-Reimers, mündliche Mitteilung). Lediglich eine ungewöhnlich hohe Oberwasserzufuhr verbesserte die Sauerstoffwerte in der Elbmündung wieder und beendete auf diese Weise das Fischsterben.

Wie die Probenanalysen zeigten, wurden einzelne Altersgruppen von Aal und Stint von dem Sterben erfaßt. Dem größeren Teil der Flundern war eine Abwanderung aus dem betroffenen Gebiet offenbar möglich, wie ihre zahlenmäßig hohe Wiederkehr in den folgenden Proben zeigte. Durch die spezifische Formation des Brackwasserkörpers im Elbe-Astuar (Maier-Reimers, in Vorbereitung) waren die von der Flunder bewohnten Bodenzonen von dem Sauerstoffdefizit wenig betroffen. Das Stintvorkommen regenerierte sich erst nach dem Auftreten der 0-Gruppe 1978 durch die Zuwanderung der I-, IIund III-Gruppen aus der Elbmündung völlig. Die Zahlen erwachsener Aale (Gelbaale) wuchsen bei Beginn der herbstlichen Gelbaal- und Blankaalwanderung wieder.

Bei der Bewertung der derzeitigen Qualität der Elbe als Lebensraum muß beachtet werden, daß die bisher vorliegenden Untersuchungen der vergangenen Jahre während einer Periode ausgesprochen hoher jährlicher Oberwasserzuflüsse durchgeführt wur- 
den, d. h. während eines Zeitraumes starker Verdünnungen der Schadstofffracht des Flusses. Derartige Sauerstoffdefizite wie im Mai 1978 könnten während der periodisch wieder zu erwartenden Niedrigwasserabflüsse (Puffahrt, 1975) zur lokalen Vernichtung einzelner Fischpopulationen des Elbe-Ästuars führen.

Danksagungen, Für Diskussionen über die Hydrologie des Elbe-Astuars danke ich Dr. E. MaierReimers, Max-Planck-Institut für Meteorologie, Hamburg, und Prof. Dr. H. Wilkens, Zoologisches Institut und Zoologisches Museum der Universität Hamburg, für die ständige Diskussionsbereitschaft. Herrn Dr. F. Hölzel, Universitäts-Krankenhaus Hamburg-Eppendorf, danke ich für die Durchsicht des Manuskripts. Außerst intensive Unterstützung erfolgte durch die Herren $H$. und E. Rübke, $\mathrm{H}$. Oessmann und $\mathrm{H}$. Oestmann (alle Fischer von Altenwerder). Der Verwaltung des Kernkraftwerkes Brunsbüttel, insbesondere Herrn Dipl.-Ing. Bajor, ist für die ständige Hilfsbereitschaft bei der Durchführung der Feldarbeiten zu danken. Der Bundesforschungsanstalt für Fischerei (Dr. G. Rauck) sei für die gelegentlich erfolgte Unterstützung der Probenahmen mein Dank ausgesprochen. - Diese Ergebnisse sind Teil einer Dissertation, die im Fachbereich Biologie der Universität Hamburg angefertigt wird.

\section{ZITIERTE LITERATUR}

Aker, E. \& Koops, H., 1970. Das Wanderverhalten der Aale in der Nordsee und die Befischungsintensität. - InfnFischw. Auslds 17, 46.

Aker, E. \& Koops, H., 1973. Untersuchungen über die Aalbestände in der Deutschen Bucht. - Arch. FischWiss. 24, 19-39.

ARGE Elbe, 1977, 1978, 1979. Wassergütedaten der Elbe, 44-84.

Baggermann, B, 1957. An experimental study on the timing of breeding and migration in the threespined stickleback (Gasterosteus aculeatus L.). - Symp. Soc. exp. Biol. 20, 105-317.

Bauch, G., 1958. Untersuchungen über die Gründe des Ertragsrückganges der Elbfischerei zwischen Elbesandsteingebirge und Boizenburg. - Z. Fisch. 7, 161-437.

Benda, R. S., Gulvas, J. \& Neal, T., 1974. Effect of power plant passage on algal primary productivity. - Proc. Indiana Acad. Sci. 84, 85-88.

Brinkmeyer, U., 1978. Die Ei-, Larval- und Jungfischentwicklung beim Stint in der Elbe im Vergleich zur Situation von 1957 bis 1959. Dipl-Arb., Univ. Hamburg, 57 pp.

Capuzzo, J. M., 1980. Impact of power plant discharges on marine zooplancton: A review of thermal, mechanical and biocidal effects. - Helgoländer Meeresunters, 33, 401-414.

Donze, M., 1979. Measurements on the effect of heat shocks on survival and growth of natural zooplancton populations. - Hydrobiol. Bull. 13, 97.

Ehrenbaum, E., 1896. Beiträge zur Naturgeschichte einiger Elbfische. - Wiss. Meeresunters. (Helgoland) 1, 54-63.

Gaudy, R. \& Moatti, B., 1979. Study of the mortality of zooplancton transient in the cooling water of a thermoelectric power plant. I.C.S.E.M., Monaco, 399-403.

Hettler, W. F. \& Clements, L. C., 1978. Effects of acute thermal stress on marine fish embryos and larvae. In: Fourth national workshop on entrainment and impingement. EA Communications, Melville, New York, 171-190.

Hynes, H. B. N., 1972. The ecology of running waters, Liverpool Univ., Liverpool, $555 \mathrm{pp}$.

Iman, A. K. E. S., Lühmann, M. \& Mann, H., 1958. Über Neunaugen und Neunaugenfischerei in der Elbe. - Fischwirt. 8, 249-260.

Kellogg, R. L., Salerno, J. J. \& Latimer, D. L., 1977. Effects of acute and chronic thermal exposures on the eggs of three Hudson River anadromous fishes. - DOE Symp. Ser. 48, 714-725.

Köhler, A., 1979. Pathologische Veränderungen der inneren Organe von Flunder (Platichthys flesus L.) und Stint (Osmerus eperlanus L.) in der Unterelbe. - Verh. dt. zool. Ges. 72, 245.

Köhler, A., 1980. Vorkommen und Gesundheitszustand von Flunder (Platichthys flesus L.) und Stint (Osmerus eperlanus L.) in der Unterelbe im Jahre 1978. Dipl.-Arb. Univ., Hamburg, 71 pp.

Köhler, A. \& Hölzel, F., 1980. Investigation on health conditions of flounder and smelt in the Elbe estuary. - Helgoländer Meeresunters. 33, 401-414. 
Lillelund, K., 1961. Untersuchungen über die Biologie und Populationsdynamik des Stintes, Osmerus eperlanus (L. 1758) der Elbe. - Arch. FischWiss. 12, 1-128.

Lucht, F., 1965. Hydrographie des Elbe-Ästuars. - Arch. Hydrobiol. (Suppl.) 29, 1-96.

Mann, H., 1963. Beobachtungen über den Aalaufstieg in der Aalleiter an der Staustufe Geesthacht im Jahre 1961. - Fischwirt 13, 182-186.

Mann, H., 1965. Über das Rückkehrvermögen verpflanzter Flußaale. - Arch. FischWiss. 15, $177-185$.

Münzing, J., 1959. Biologie, Variabilität und Genetik von Gasterosteus aculeatus L. (Pisces). Untersuchungen im Elbegebiet. - Int. Revue ges. Hydrobiol. 44, 317-382.

Münzing, J., 1962. Die Populationen der marinen Wanderformen von Gasterosteus aculeatus L. (Pisces) an den holländischen und deutschen Nordseeküsten. - Neth. J. Sea Res. 1, 508-525.

Münzing, J., 1963. Die Verbreitung des dreistacheligen Stichlings in Europa. - Natur Mus., Frankf. 93, 284-290.

Puffahrt, O., 1975. Allgemeine Betrachtungen über den Talsperren- und Speicherbau der CSSR und DDR im Einzugsgebiet der Elbe und die Auswirkungen auf den Hochwasser- und Niedrigwasserabfluß der Elbe. Wasserwirtschaftsamt Lüneburg, $40 \mathrm{pp}$.

Rheinheimer, G., 1971. Mikrobiologie der Gewässer. VEB Fischer, Jena, 184 pp.

Schnackenbeck, W., 1928. Die Nordseefischerei. In: Handbuch der Seefischerei. Hrsg. v. H. Lübbert \& E. Ehrenbaum. Schweizerbart, Stuttgart, 5, 37-69.

Schubel, J. R., Smith, C. F. \& Koo, T. S. Y., 1977. Thermal effects of power plant entrainment on survival of larval fishes: a laboratory assessment, - Chesapeake Sci. 18, 290-298.

Tesch, F.-W., 1967. Aktivität und Verhalten wandernder Lampetra fluviatilis, Lota lota und Anguilla anguilla im Tidegebiet der Elbe. - Helgoländer wiss. Meeresunters. 16, 92-111.

Tesch, F.-W., 1977. The eel. Chapman \& Hall, London, 434 pp.

Thurow, F., 1959. Über Fangerträge und Wachstum des Aales in der westlichen Ostsee. - Z. Fisch. 8, 597-626.

Tiews, K., 1960. Untersuchungen über die Winterfischerei auf Hering (Spitzen) und Sprott im Mündungsgebiet der Weser und Ems in den Jahren 1958/59 und 1959/60 - Arch. FischWiss. 12, 75-100.

Tiews, K., 1971. Weitere Ergebnisse von Langzeitbeobachtungen über das Auftreten von Beifangfischen und -krebsen in den Fängen der deutschen Garnelenfischerei (1961-1967). - Arch. FischWiss. 22, 214-255.

Wilkens, H. \& Köhler, A., 1977. Die Fischfauna der unteren und mittleren Elbe: die genutzten Arten, 1950-1975. - Abh. Verh. naturw. Ver. Hamburg 20, 185-222.

Wootton, R. J., 1976. The biology of the sticklebacks. Acad. Press, London, 387 pp. 